\title{
A microfabricated photonic magnetometer
}

\author{
Jan Preusser, Svenja Knappe, John Kitching \\ Time and Frequency Division \\ NIST \\ Boulder, CO, USA \\ knappe@boulder.nist.gov
}

\begin{abstract}
We present initial measurements of an atomic magnetometer in which the microfabricated sensor head is coupled to the control system by optical fibers. Because there are no electrical connections, the sensor head is largely nonmagnetic, and degradation of the magnetometer performance due to internal magnetic fields is avoided. The sensor head is fabricated by use of methods derived from microelectromechanical (MEMS) systems and integrated with off-theshelf micro-optical components. Light is coupled through fibers to and from the sensor head for interrogation of the atoms as well as temperature stabilization of the vapor cell.
\end{abstract}

\section{INTRODUCTION}

Over the past five years, our group at NIST has developed small atomic magnetometers that are fabricated with methods derived from microelectromechanical systems (MEMS). In most cases, these magnetometers rely on precise frequency measurements of magnetically sensitive atomic transitions of alkali atoms in vapor cells. Low-power diode lasers optically pump the atoms and create a magnetic polarization. They can then precess in a magnetic field at the Larmor frequency, and the precession can be detected as a modulation on a probe laser beam [1]. To miniaturize atomic magnetometers, MEMS techniques are used to fabricate the alkali vapor cells [2], cell heaters, and optical components. These components are integrated with low-power diode lasers and photodiodes into physics packages near $10 \mathrm{~mm}^{3}$ in size.

The first chip-scale atomic magnetometer (CSAM) was based on coherent population trapping (CPT) spectroscopy [3]. A sensitivity of $50 \mathrm{pT} / \sqrt{\mathrm{Hz}}$ in an active volume of $1 \mathrm{~mm}^{3}$ was measured (see blue trace A in Figure 1). The large bandwidth of $13 \mathrm{kHz}$ and low intrinsic heading errors of CPT magnetometers, as well as the fact that they can be driven as scalar sensors without dead zones, could make them suitable for certain mobile applications, such as geophysical surveys. Better sensitivities in earth magnetic field could be reached with so-called Mx magnetometers [4] that measure Zeeman rather than hyperfine resonances (red trace B in Figure 1). A sensitivity of $5 \mathrm{pT} / \sqrt{\mathrm{Hz}}$ was reached [5]. In the $\mathrm{Mx}$

\author{
Vladislav Gerginov \\ PTB \\ Braunschweig, Germany
}

magnetometer in [5], the resonance linewidth of roughly 1 $\mathrm{kHz}$ was dominated by $\mathrm{Rb}-\mathrm{Rb}$ spin-exchange collisions. The broadening due to these collisions can be suppressed [6] when the spin-exchange collision rate is much larger than the Larmor precession frequency of the atoms. A magnetometer operating in this spin-exchange relaxation-free (SERF) [7] regime based on a MEMS rubidium cell was constructed with a simple one-beam geometry [8]. At a cell temperature of 150 ${ }^{\circ} \mathrm{C}$, the cell temperature is high enough that the linewidth narrows substantially in a very low magnetic field compared to that in the spin-exchange limited case. A sensitivity of 70 $\mathrm{fT} / \sqrt{ } \mathrm{Hz}$ was reached for the zero-field resonance (green trace $\mathrm{C}$ in Figure 1) with a bandwidth of $580 \mathrm{~Hz}$.

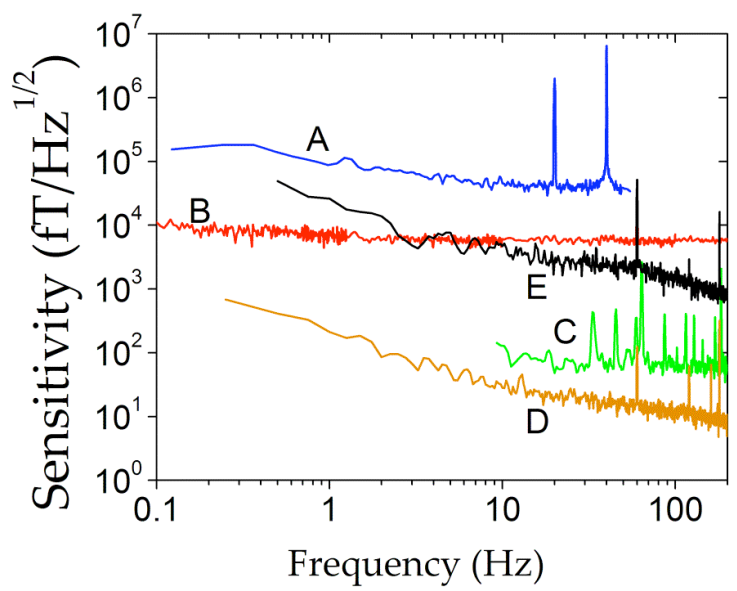

Figure 1. Magnetic field sensitivity of microfabricated atomic magnetometers at NIST as a function of frequency. Trace A (blue): CPT CSAC [3], trace B (red): Mx CSAC [5], trace C (green) SERF magnetometer [8], trace D (orange) Flux concentrator magnetometer [9], trace E (black): Photonic Bell-Bloom magnetometer

The magnetometer in [8] operated near zero field and was therefore most useful in a magnetically shielded environment as is found for example in certain medical applications. Even better sensitivities can be reached when the miniature vapor cells are integrated with flux concentrators. The concentrator 
noise then limits the sensitivity to $10 \mathrm{fT} / \sqrt{ } \mathrm{Hz}$ [9] (see orange trace D in Figure 1).

\section{PHOTONIC MAGNETOMETER DESIGN}

When miniaturizing these sensitive magnetometers, magnetic fields produced by the sensors themselves have to be minimized [10]. Due to the small distances, tiny currents flowing in such components as laser, photodetector, RF coils, or cell heaters can produce magnetic fields at the location of the vapor cell large enough to cause reduced sensitivity or heading errors (reading errors due to the orientation of the magnetic field relative to the sensor). Furthermore, in arrays of many sensors, magnetic fields produced by one sensor can interfere with the measurement of the surrounding ones.

In this paper, we introduce a magnetometer design that eliminates such fields by removing all conducting surfaces far from the sensor volume. Magnetometer interrogation and temperature control of the sensor are performed solely by light brought to and from the vapor cell by means of optical fibers. The spin-precession of the atoms is driven by a modulated optical field [1]. The design of the sensor head is shown schematically in Figure 2. The ${ }^{87} \mathrm{Rb}$ atoms are confined in a $1 \mathrm{~mm}^{3}$ volume inside a MEMS vapor cell [2]. The $795 \mathrm{~nm}$ pump light is transported to the sensor head through a polarization-maintaining single-mode fiber $5 \mathrm{~m}$ long.

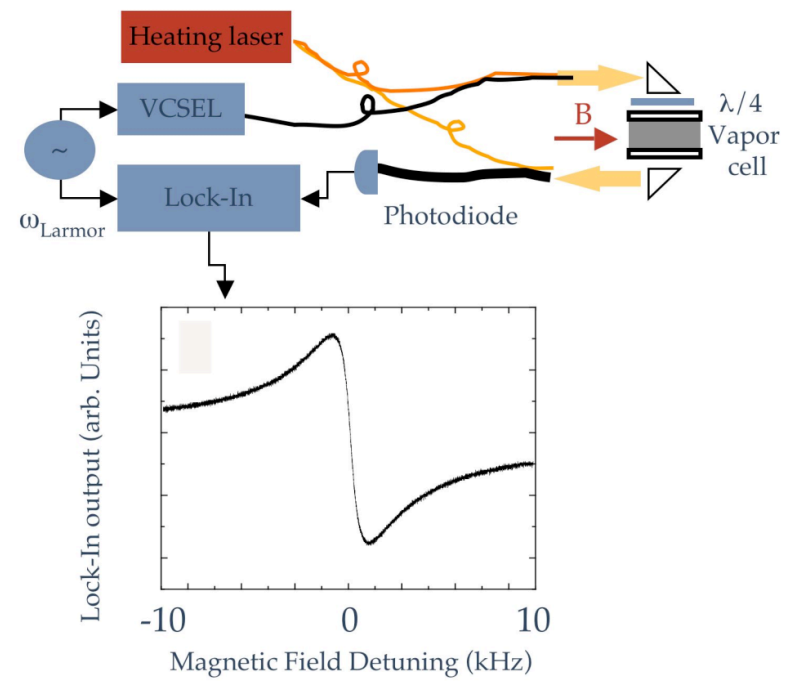

Figure 2. Schematic of the photonic magnetometer setup operated in BellBloom configuration. The light from the pump laser is delivered to the vapor cell by optical fibers

A microprism reflects the beam at a right angle so that it passes through a quarter-wave plate and the ${ }^{87} \mathrm{Rb}$ vapor cell (see Figure 3a). A second microprism reflects the beam onto a multimode fiber of $1 \mathrm{~mm}$ diameter that leads the light to a photodetector placed far away from the vapor cell. The fibers and optical components are fit tightly into $1 \mathrm{~mm}$ thick silicon supports fabricated by deep reactive ion etching (DRIE). Five supports are stacked on top of each other and form the enclosure of the sensor head.

The vapor cell must be heated to roughly $90{ }^{\circ} \mathrm{C}$ in order to increase the vapor pressure enough to optimize the magnetometer signal. This is done by light from a diode laser at a wavelength of $915 \mathrm{~nm}$. Two multimode fibers run parallel to the pump and detection fibers, and to both sides of the sensor head. The light reflected thought the two microprisms is absorbed by the silicon rim of the vapor cell and ensures that the windows are kept slightly hotter than the cell body to minimize the condensation of alkali atoms on the window surfaces. To increase the absorption of the silicon rims, they are painted black at the locations of light incidence.

The vapor cell is suspended in the silicon enclosure by two webs of polyimide tethers that are attached to both sides of a silicon supports [11], as shown in Figure $3 \mathrm{~b}$. The conductive thermal losses when maintaining a temperature difference of $80{ }^{\circ} \mathrm{C}$ were estimated to be roughly $1 \mathrm{~mW}$. In the future, the sensor head enclosure will be evacuated, so that the power dissipation will be limited by radiative losses. Assuming blackbody radiation, a total power consumption of $25 \mathrm{~mW}$ can be estimated.

(a)

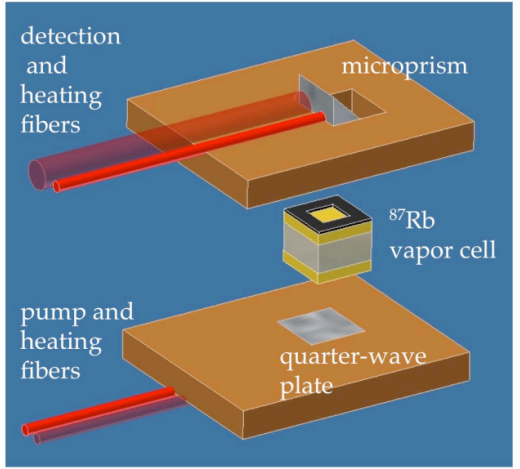

(b)

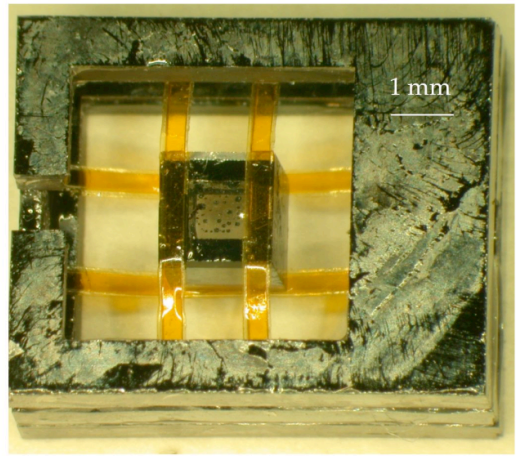

Figure 3. (a) Schematic of the photonic magnetometer sensor head. (b) Photograph of the sensor head interior. The vapor cell (active volume $1 \mathrm{~mm}^{3}$ ) is suspended on a polyimide web that rests on a silicon rim.

The "photonic" magnetometer can be operated in different modes, depending on the desired application. In earth magnetic field, the sensor can be driven in the so-called Bell- 
Bloom mode, where the polarization of the atoms is driven at the Larmor frequency by modulating the laser frequency on and off the atomic resonance [1]. When comparing the phase of the modulated signal detected at the Larmor frequency with that driving the laser, a resonance of the atomic magnetization can be detected as the modulation frequency is scanned through the Larmor resonance. A schematic of this setups and a picture of such a resonance are shown in Figure 2. The laser frequency is stabilized onto the atomic resonance by use of a separate evacuated $\mathrm{Rb}$ vapor cell. This external spectroscopy, as well as pump and heating lasers and photodetectors, are placed far from the sensor head. The sensor head is placed inside a single-layer magnetic shield, and a magnetic field of $7 \mu \mathrm{T}$ is applied perpendicular to the light beam. The laser frequency is modulated close to the Larmor frequency. Phase-sensitive detection is used to monitor the modulation on the photodetector. Such a signal, detected with a lock-in amplifier when the laser modulation frequency is swept around $50 \mathrm{kHz}$ is shown in Figure 2.

The sensitivity of this magnetometer can be seen in Figure 1 (black trace E). The sensitivity of $2.6 \mathrm{pT} / \sqrt{\mathrm{Hz}}$ is comparable to that if the Mx CSAM [4]. We are therefore confident that our design can perform similarly to previous CSAMs. In the future, we plan to operate this sensor in the SERF regime at low magnetic fields with higher magnetic sensitivity. In order to make these devices useful for many applications, arrays of sensors will be driven by one supply laser system that allows for gradiometer measurements.

\section{CONCLUSIONS}

We have introduced a first prototype of a photonic magnetometer sensor head. To minimize interference from magnetic fields produced by the sensor heads themselves, the prototype was fabricated without metallic objects near the active volume. Current-carrying components such as the laser, photodetector, and heaters are placed far from the active volume of the magnetometer, and the magnetometer is pumped, probed, and temperature stabilized with light, carried to the atoms through optical fibers. The sensor head design is made by use of MEMS bulk etching and bonding methods that are integrated with micro-optics components. The sensitivities measured with the photonic magnetometer are similar to those achieved with comparable magnetometers based on previous chip-scale designs.

\section{ACKNOWLEDGEMENTS}

The authors thank S. Schima for help with the sensor head fabrication. This work is a partial contribution of NIST, an agency of the US government, and is not subject to copyright.

\section{REFERENCES}

[1] W. Bell and A. Bloom, "Optical Detection of Magnetic Resonance in Alkali Metal Vapor", Phys. Rev. vol. 10, p. 1559-1565,1957.

[2] L.-A. Liew, S. Knappe, J. Moreland, H. Robinson, L. Hollberg, K. Kitching, "Microfabricated alkali atom vapor cells," Appl. Phys. Lett. Vol. 84, pp. 2694-2696, 2004.

[3] P. D. D. Schwindt, S. Knappe, V. Shah L. Hollberg, K. Kitching L.-A. Liew, J. Moreland "Chip-scale atomic magnetometer," Appl. Phys. Lett., vol. 85, pp. 6409-6411, 2004.

[4] A. Bloom, "Principles of Operation of the Rubidium Vapor Magnetometer," Appl. Opt., vol. 1, pp. 61-68, 1962

[5] P. D. D. Schwindt, B. Lindseth, S. Knappe, V. J. Kitching, "A chipscale atomic magnetometer with improved sensitivity using the $\mathrm{Mx}$ technique," Appl. Phys. Lett., vol. 90, pp. 081102, 2007.

[6] W. Happer and A. C. Tam, "Effect of rapid spin exchange on the magnetic-resonance spectrum of alkali vapors," Phys. Rev. A, vol. 16, pp. 1877-1891, 1977.

[7] J. C. Allred, R. N. Lyman, T. W. Kornack, M.V. Romalis, "Highsensitivity atomic magnetometer unaffected by spin-exchange relaxation," Phys. Rev. Lett., vol. 89, pp. 130801, 2002.

[8] V. Shah, S. Knapppe, P. Schwindt, J. Kitching, "Subpicotesla atomic magnetometry with a microfabricated vapour cell," Nat. Phot., vol. 1, pp. 649-652, 2007.

[9] W.C. Griffith, R. Jimenez-Martinez, V. Shah, S. Knappe, and J. Kitching. "Miniature atomic magnetometer integrated with flux concentrators,"Appl. Phys. Lett., vol. 94, pp. 123502, 2009.

[10] J. Belfi, G. Bevilacqua, V. Biancalana, Y. Dancheva, L. Moi, "All optical sensor for automated magnetometry based on coherent population trapping", J. Opt. Soc. Am. B, vol. 24, p. 1482-1489, 2007.

[11] M. Mescher, R. Lutwak, R. Varghese "An ultra-low-power physics package for a chip-scale atomic clock," IEEE Solid-State Sensors and Actuators 2005. 